\title{
Concurrent biological targeting therapy of squamous cell carcinoma of the esophagus with cetuximab and trastuzumab
}

\author{
MASANAO YAMAZAKI, YOSHITO YAMASHITA, NAOSHI KUBO, \\ MASAKAZU YASHIRO, MASAICHI OHIRA, EIJI AKO, HIROAKI TANAKA, \\ KAZUYA MUGURUMA, TETSUJI SAWADA and KOSEI HIRAKAWA
}

Department of Surgical Oncology, Graduate School of Medicine, Osaka City University, Osaka 545-8585, Japan

Received February 24, 2012; Accepted April 10, 2012

DOI: $10.3892 /$ or.2012.1803

\begin{abstract}
The ERBB proteins are cell membrane tyrosine kinase receptors. Among these receptors, ERBB1 (EGFR or HER1) and ERBB2 (HER2/Neu) have been reported to be the most important in terms of the development and progression of squamous cell carcinoma of the esophagus (SCC). Thus, targeting of ERBB1 and ERBB2 may become a promising strategy to treat SCC. In the present study, we examined ERBB1 and ERBB2 expression of SCC cell lines (TT, TE2, TE6 and TE10) and tumor samples. In addition, we evaluated the effect of the anti-ERBB1 antibody cetuximab and the antiERBB2 antibody trastuzumab for SCC in vitro and in vivo. Biological activities (receptor downregulation, the phosphorylation of MAPK and AKT) induced by the two agents were also investigated. Immunohistochemistry of SCC samples showed that ERBB1 was detected in 84\%, while ERBB2 was detected in $30 \%$. RT-PCR analysis revealed that ERBB1 and ERBB2 mRNA were detectable in all four cell lines. MTT cell proliferation analysis showed that cetuximab, but not trastuzumab, inhibited growth in each of the SCC cell lines in a dose-dependent manner. Further, cetuximab and trastuzumab used together produced stronger inhibition of growth compared to cetuximab alone. Cetuximab downregulated ERBB1, but not ERBB2, at the TE6 cell surface. However, neither ERBB1 nor ERBB2 showed any downregulation by trastuzumab at the TE6 cell surface. Cetuximab, not but trastuzumab, inhibited the phosphorylation of MAPK and Akt. When administered in combination, the two agents inhibited Akt phosphorylation to a greater degree compared to treatment with cetuximab alone. In the in vivo study, cetuximab, but not trastuzumab, significantly inhibited the TT tumors. Additionally, the combination of cetuximab with trastuzumab induced a synergistic inhibitory
\end{abstract}

Correspondence to: Dr Naoshi Kubo, Department of Surgical Oncology, Graduate School of Medicine, Osaka City University, 1-4-3 Asahimachi, Abeno-ku, Osaka 545-8585, Japan

E-mail: k-naoshi@med.osaka-cu.ac.jp

Key words: esophageal cancer, ERBB1, ERBB2, cetuximab, trastuzumab antitumor effect in the TT tumors. In conclusion, combination of cetuximab and trastuzumab revealed a synergistic antitumor effect for SCC in vitro and in vivo. The antitumor effect may be induced by the inhibition of the phosphorylation of Akt. These findings suggest that combination therapy including cetuximab and trastuzumab may be a promising strategy to treat SCC.

\section{Introduction}

Esophageal cancer (EC) is one of the most lethal cancers in Western countries as well as in Japan. Most patients with esophageal cancer in Japan have squamous cell carcinoma, while most of those in Western countries have adenocarcinoma. By the time it is diagnosed, the cancer has often progressed to such an advanced stage that $>50 \%$ of patients show signs of metastasis or unresectable invasion (1). Despite the improvements of various treatment modalities such as surgical resection, chemotherapy and chemoradiotherapy, the prognosis of patients with EC has remained poor. It has been reported that recent overall 5-year survival rate for EC patients was $15-42 \%(2,3)$.

Within this context it has been proposed that novel approaches involving targeted biological therapies may provide an additional therapeutic benefit. Although a number of clinical trials involving a variety of biological therapies are now ongoing $(4,5)$, numerous questions remain surrounding the mechanisms of action of these agents both in esophageal cancer specifically as well as within various cancer types.

The ERBB protein family consisting of ERBB1 (also known as EGFR or HER1), ERBB2 (HER2/Neu), ERBB3 (HER3) and ERBB4 (HER4), are cell membrane tyrosine kinase receptors which play key roles in cancer development and progression. ERBB receptors are activated by the formation of homo- or heterodimers between different members of the ERBB receptor family in response to ligand binding (6-9). ERBB2 and ERBB3 are thought to only form heterodimers and are considered non-autonomous receptors, ERBB2 has no known direct ligand, although it is considered the preferred heterodimerization partner and a signal amplifier and ERBB3 lacks intrinsic kinase activity (6-10). Following dimerization, ERBB receptor phosphorylation results in an intracellular cascade of downstream substrate 
activations which in turn induce mitogenic signaling and other cellular activities. Two major signaling routes activated by ERBB receptor family members are the Ras-Raf-MAPkinase pathway and a pathway that includes PI3-kinase and the downstream protein kinase Akt (6,8,9,11-13).

Numerous cancer cell types including EC cells, express high levels of growth factor and growth factor receptors and exhibit autocrine- or paracrine-stimulated growth $(8,14)$. Of the four members of the receptor superfamily, ERBB1 and ERBB2 appear to be the most important with regards to EC (14-17). Numerous studies conducted in EC patients and cell lines have reported overexpression of ERBB1 and ERBB2 as well as a correlation between ERBB1 and ERBB2 expression and prognosis, although some contrary findings have also been reported (13-20). Given the important role ERBB receptor signaling may play in the progression of $\mathrm{EC}$, it has been reasoned that blocking ERBB1 and ERBB2 might prove an effective strategy for cancer treatment $(14,16,18-20)$. Using two well-known antiERBB receptor monoclonal antibodies, we sought to further investigate their inhibitory effects on EC cell lines and elucidate some potential mechanisms by which this might occur.

Cetuximab (Erbitux ${ }^{\mathrm{TM}}$ ) is an anti-ERBB1 monoclonal antibody approved for clinical use in colorectal cancers and is in trials for EC among others $(15,21,22)$. Cetuximab is thought to act via a variety of mechanisms including blockage of ligand binding and receptor dimerization, receptor downregulation, inhibition of the cell cycle and cellular proliferation, apoptosis induction, sensitization to chemotherapy and via the induction of antibody-dependent cell-mediated cytotoxicity (ADCC) $(21,22)$. Trastuzumab (Herceptin $\left.{ }^{\mathrm{TM}}\right)$, an anti-ERBB2 monoclonal antibody, is used in the treatment of patients with ERBB2-overexpressing breast cancer and, like cetuximab, is in trials for EC amongst others $(15,21,22)$. Trastuzumab is also thought to act via a variety of mechanisms including ADCCmediated activity, blockage of receptor hetero-dimerization, prevention of ectodomain shedding, and angiogenesis inhibition, whereas its role with regard to receptor downregulation remains controversial $(21,22)$. Moreover, although the effects of these agents on receptor downregulation and intracellular signaling pathways has been investigated in a number of other cancers, to our knowledge the mechanisms by which cetuximab and trastuzumab affect these processes in EC remain largely unexamined.

Here, we first sought to establish the level of expression of ERBB1 and ERBB2 in several EC cell lines. We then evaluated the effect of cetuximab and trastuzumab, both alone and in combination on cellular proliferation. Further, we investigated how cetuximab and trastuzumab alone and in combination exert their anti-carcinogenic effect in these cell lines, namely via receptor downregulation and inhibition of downstream kinase activity. Finally, we examined the effects of these agents on the growth of EC xenograft tumors in vivo.

\section{Materials and methods}

Cell culture and tumor samples. TT, TE2, TE6 and TE10 human esophageal squamous cell carcinoma cells were obtained from the Health Science Research Resources Bank (Osaka, Japan) and the Department of Advanced Surgical Science and Technology of Tohoku University Graduate
School of Medicine (Sendai, Japan). Cells were cultured in Dulbecco's modified Eagle's medium (DMEM; Bioproducts, Walkersville, MD, USA), fetal bovine serum (FBS; Gibco, Grand Island, NY, USA), penicillin and streptomycin (ICN Biomedicals, Inc., Costa Mesa, CA, USA) and sodium pyruvate (Bioproducts).

With regard to tumor samples, 50 cases with primary esophageal squamous cell carcinoma that underwent surgical resection at the Department of Surgical Oncology, Osaka City University Hospital between 2005 and 2007, were examined. All patients had no preoperative chemotherapy and chemoradiotherapy. The study was approved by the Ethics Committee of the Osaka City University, and informed consent was obtained from all patients. Tumor tissue were fixed in $4 \%$ buffered formalin, dehydrated and embedded in paraffin. Sections, $4-\mu \mathrm{m}$ thick were cut and deparaffinized in xylene and hydrated through ethanol to distilled water.

Animal care. Female athymic BALB/c nude mice, 4 weeks of age, were purchased from Crea Japan, Inc. (Tokyo, Japan). The mice were kept in sterilized cages equipped with an air filter and sterile bedding materials, and provided sterilized food and water throughout the study. All procedures were performed in accordance with Osaka City University Medical School's ethical guidelines for animal experiments.

Chemotherapeutic antibody treatment. The human-mouse chimeric anti-ERBB1 monoclonal antibody cetuximab was supplied by ImClone Systems, Inc. (New York, NY, USA) and the humanized anti-ERBB2 monoclonal antibody trastuzumab (Herceptin) by Nippon Roche K.K. (Tokyo, Japan).

Immunohistochemistry. Immunostaining was conducted by the avidin-biotin method using a Histofine kit (Nichirei Co., Ltd., Tokyo, Japan). Sections were incubated overnight at $4^{\circ} \mathrm{C}$ with undiluted anti-ERBB1 antibody (Zymed Laboratory, Inc., San Francisco, CA, USA) or for $60 \mathrm{~min}$ at room temperature with anti-ERBB2 antibody diluted at 1:800 (Nichirei Co., Ltd.). For immunohistochemical evaluation of ERBB1 and ERBB2, cells were treated as positive when immunoreactivity was clearly observable using the widely-employed 0-3+ scoring system to assign relative values.

Reverse transcription-polymerase chain reaction. Human ERBB1 and ERBB2 primer kits (Maxim Biotech, Inc., San Francisco, CA, USA) were used to confirm ERBB1 and ERBB2 expression in EC cells. RNA was extracted using TRIzol (Life Technologies, Inc., Gaithersburg, MD, USA) following the manufacturer's instructions. Total DNA was reverse-transcripted from a reaction buffer containing oligo(dT) primer, RNA PCR buffer, $10 \mathrm{mM}$ dNTPs, $0.1 \mathrm{M}$ DTT, RNA guard (Amersham Pharmacia Biotech, Inc., Buckinghamshire, UK) and Moloney murine leukemia virus reverse transcriptase (Life Technologies, Inc.). cDNA samples were amplified from a PCR reaction mixture containing each primer set and Taq-polymerase (AmpliTaq Gold, Applied Biosystems Japan, Ltd., Tokyo, Japan). The primers used were 5'-CGTTGCTGGCTGCGCTCTG-3' and 5'-AGCCACCTCCTGGATGGTC-3' for ERBB1 (product size, $221 \mathrm{bp}$ ) and 5'-GCTGGCTCCGATGTATTTGATGGT-3' 
$\mathbf{A}$

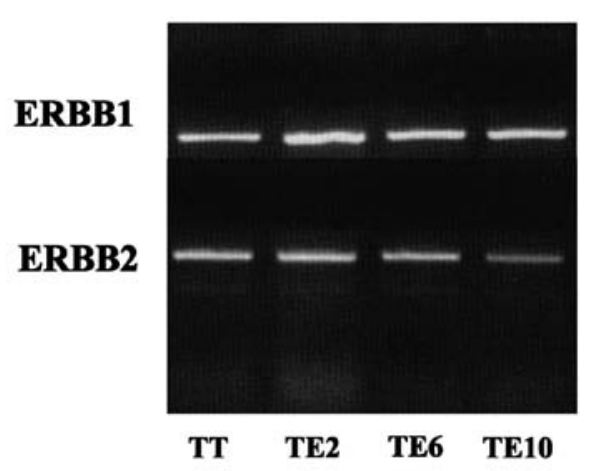

B

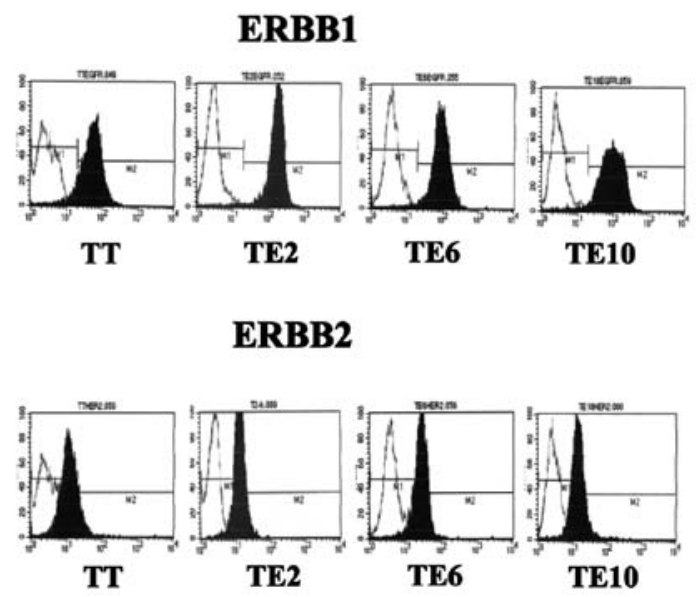

Figure 1. RT-PCR and flow cytometry analysis of ERBB1 and ERBB2 expression in EC cell lines. RT-PCR analysis showed mRNA of ERBB1 and ERBB2 was identified in all EC cell lines (A). The expression of ERBB1 and ERBB2 were found in all EC cell lines by flow cytometry analysis (B). In all EC cell lines, ERBB1 expression appeared to be higher than that of ERBB2.

and 5'-GTTCTCTGCCGTAGGTGTCCCTTT-3' for ERBB2 (product size, $495 \mathrm{bp}$ ).

Immunolabeling and FACS analysis. EC cells $\left(1 \times 10^{6}\right)$ were incubated on ice with the primary antibodies. Mouse antibodies used were anti-ERBB1 (Oncogene Research Products, Cambridge, MA, USA) and anti-ERBB2 (NeoMarkers, Inc., Fremont, CA, USA). After washing, the cells were suspended on ice in FITC-labeled goat anti-mouse IgG Fc-specific secondary antibody (Chemicon International Inc., Temecula, CA, USA). The cells were then washed again resuspended in flow buffer, and analyzed by FACS (CellQuest FACS Calibur, Becton-Dickinson \& Co., San Jose, CA, USA).

Cell proliferation assay. Inhibition of EC cell growth in vitro was determined by a [methyl- $\left.{ }^{3} \mathrm{H}\right]$-thymidine incorporation proliferation assay, in which $2 \times 10^{4}$ cells per well were plated and incubated for $24 \mathrm{~h}$ with increasing concentrations of either or both cetuximab and trastuzumab (100-1000 ng/ml). Following this treatment, $0.5 \mathrm{mCi} /$ well [methyl- ${ }^{3} \mathrm{H}$ ]-thymidine (Amersham Biosciences, Inc., Piscataway, NJ, USA) was added and the cultures were incubated for an additional $24 \mathrm{~h}$. The cells were then harvested, and the amount of incorporated radioactivity was determined by liquid scintillation counting.

Influence of cetuximab and trastuzumab on ERBB1 and ERBB2 expression. Flow cytometric analysis was used to examine the influence of cetuximab and trastuzumab on the expression of ERBB1 and ERBB2. Cells $1 \times 10^{4}$ per well were plated for $6 \mathrm{~h}$ in FBS-supplemented DMEM containing increasing concentrations of either or both cetuximab and trastuzumab $(1000 \mathrm{ng} / \mathrm{ml}$ each). ERBB1 and ERBB2 expression was analyzed using the method previously described for FACS analysis.

Phosphorylation assay. An indirect measure of kinase activity in the cells was obtained by detection of phosphorylated MAPK and Akt (p-MAPK and p-Akt) on immunoblots. Cells were incubated for $48 \mathrm{~h}$ with either or both cetuximab or trastuzumab (1000 ng/ml), lysed by adding 1X SDS sample buffer and immediately scraped from the plate and transferred with the extract to a microcentrifuge tube, in which they were sonicated for 10-15 sec to shear DNA and reduce sample viscosity. A $20-\mu 1$ sample of each cell lysate was heated to $95^{\circ} \mathrm{C}$ for $5 \mathrm{~min}$ and placed on ice. After microcentrifugation for $5 \mathrm{~min}, 20 \mu \mathrm{l}$ of each sample was then separated on a $12.5 \%$ SDS-PAGE and transferred to a polyvinylidene difluoride membrane. The membrane was blocked in 5\% non-fat dry milk in PBS incubated with primary antibodies, which included 1:1000 diluted anti-p-MAPK and anti-p-Akt (ser 473) as well as anti-MAPK and anti-Akt antibodies as a control (Cell Signaling Technology, Inc., Beverly, MA, USA), then incubated overnight. The next day, the membrane was incubated in peroxidase-conjugated donkey anti-rabbit IgG secondary antisera (Cell Signaling Technology; diluted 1:1500 in PBS, 5\% non-fat dry milk). Antibody binding was detected by ECL (Amersham Biosciences, Inc.) and subsequently exposed to film.

Treatment of xenografts in athymic nude mice. Nude mice were administered a subcutaneous injection of TT cells. Approximately 6 weeks following the injection, the tumor (1 $\mathrm{cm}$ in diameter) was aseptically resected and minced into $3 \times 3 \times 3 \mathrm{~mm}$ pieces. Host mice were then anesthetized with diethyl ether, and a piece of tumor was implanted into the left flank of each mouse. The tumors were allowed to reach $10-15 \mathrm{~mm}^{3}$ in size, occurring at around 7 days after implantation, at which point the mice were randomly divided into four groups of six animals each. Three groups received an intraperitoneal injection of $1 \mathrm{mg} /$ dose of cetuximab and trastuzumab, either alone or in combination, twice a week for 5 weeks. A fourth control group received intraperitoneal injection of an equivalent volume of human IgG1 twice a week for 5 weeks. Tumor volume was calculated using the following formula: tumor volume $=\left(\right.$ length $\mathrm{x}$ width $\left.{ }^{2}\right) / 2$, where length and width are the longest and shortest dimensions of the tumor, respectively. 

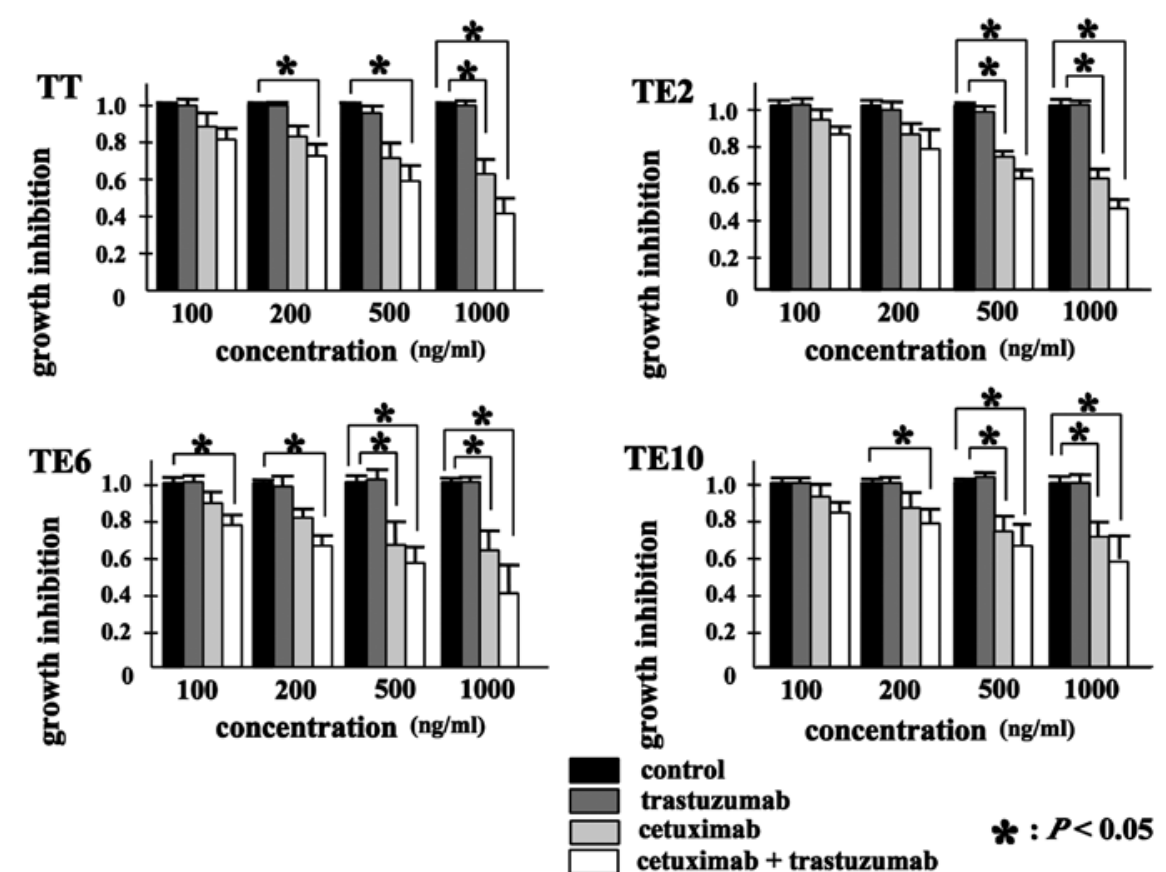

Figure 2. The growth inhibition of EC cell lines treated by cetuximab and/or trastuzumab. All EC cell lines were analyzed by MTT assay in various dose combinations of cetuximab and/or trastuzumab. Cetuximab alone showed significant anti-proliferative effects for all EC cell lines, whereas trastuzumab showed no anti-proliferative effect. However, combination of cetuximab and trastuzumab revealed synergistic anti-proliferative effect.

Statistical analysis. Comparisons among the data sets were made with an analysis of variance, followed by Student's t-test. Differences were considered to be statistically significant when the P-value was $<0.05$.

\section{Results}

Expression of ERBB1 and ERBB2. Immunohistochemistry showed ERBB1 was detected in all 50 squamous EC samples, with a staining intensity of $3+$ or $2+$ in $84 \%$, while ERBB2 was detected in 45 squamous EC samples, with an intensity of $3+$ or $2+$ in $30 \%$.

ERBB1 and ERBB2 expression in EC cells. RT-PCR analysis revealed ERBB1 and ERBB2 mRNA were detectable in all four cell lines (Fig. 1A). Flow cytometry analysis showed almost the same result as RT-PCR (Fig. 1B). Overall, ERBB1 expression appeared to be higher than that of ERBB2.

Inhibition of EC cell proliferation by cetuximab and trastuzumab in vitro. Results showed that cetuximab, but not trastuzumab, inhibited growth in each of the EC cell lines tested in a dose-dependent manner, as measured by incorporation of $\left[{ }^{3} \mathrm{H}\right]$-thymidine into DNA. Further, cetuximab and trastuzumab used together produced stronger inhibition of growth than cetuximab alone (Fig. 2). The combination of cetuximab with trastuzumab induced a synergistic inhibitory effect in all the EC cell lines.

Downregulation of ERBBI expression by cetuximab. Cetuximab downregulated ERBB1, but not ERBB2 at the TE6 cell surface. Neither ERBB1 nor ERBB2 showed any downregulation by trastuzumab at the TE6 cell surface in the present study (Fig. 3).
Expression of ERBB1

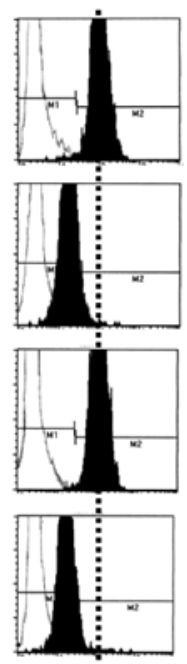

Expression of ERBB2

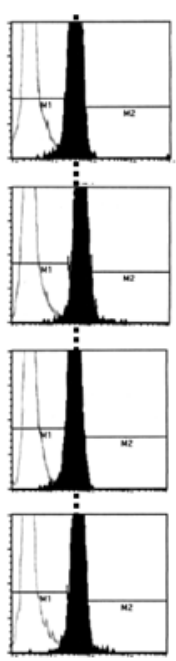

Figure 3. The expression of ERBB1 and ERBB2 at the TE6 cell surface treated by cetuximab and/or trastuzumab. Cetuximab downregulated ERBB1, while trastuzumab did not downregulate ERBB2. Additionally, downregulation of ERBB1 by the combination cetuximab and trastuzumab was seen to the same degree as by cetuximab alone.

In addition, downregulation of ERBB1 by the combination cetuximab with trastuzumab was seen to the same degree as by cetuximab alone. Similar results were obtained in the other EC cell lines.

Effect of cetuximab treatment on the Ras/Raf/MAP kinase and PI3 kinase/Akt pathways. Cetuximab inhibited the phosphorylation of MAPK and Akt (Fig. 4), whereas trastuzumab 


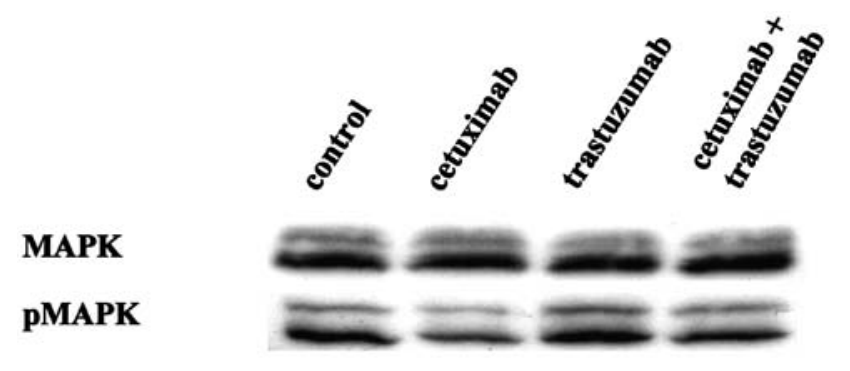

AKT

pAKT

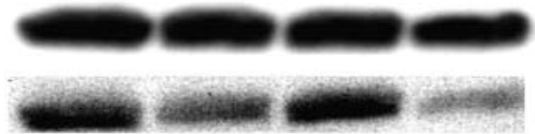

Figure 4. Inhibition of the phosphorylation of MAPK and Akt by cetuximab and/or trastuzumab. Cetuximab, but not trastuzumab, inhibited the phosphorylation of MAPK and Akt. Moreover, combination of cetuximab with trastuzumab inhibited the phosphorylation of Akt to a greater extent than cetuximab alone.

had no detectable effect on the phosphorylation states of either MAPK or Akt. When administered in combination, however, the two agents inhibited Akt phosphorylation to a greater degree than treatment with cetuximab alone.

Growth inhibition by cetuximab and trastuzumab in vivo. No mouse experienced a loss in body weight or dermatosis, and none died during the study period. Mice treated with cetuximab displayed a significant decrease in the volume of TT tumors compared to mice treated with human IgG1 $(\mathrm{P}<0.01)$, whereas trastuzumab alone could not inhibit the growth of TT tumors. However, the combination of cetuximab with trastuzumab induced a synergistic inhibitory antitumor effect in TT tumors (Fig. 5).

\section{Discussion}

In this study, we found that cetuximab downregulated ERBB1 and inhibited the phosphorylation of both the MAPK and Akt pathways in a group of EC cell lines. In contrast, trastuzumab did not downregulate ERBB2 or inhibit the phosphorylation of either the MAPK or Akt pathway. Interestingly, however, the combination of cetuximab and trastuzumab downregulated ERBB1 but not ERBB2 and inhibited the phosphorylation of the Akt pathway to a greater extent than cetuximab alone. We also showed patterns of ERBB1 and ERBB2 expression broadly similar to those in previous studies, and that cetuximab and trastuzumab, either alone or in combination, both inhibited cellular proliferation in these EC cells. These findings support a possible role for cetuximab and trastuzumab combination therapy in the treatment of EC, and may help further understanding of the complex interactions and downstream signaling of ERBB receptors in EC.

A number of review articles have reported on the variability of ERBB-receptor expression levels in EC $(13-15,18,17)$. Here, we found ERBB1 and ERBB2 overexpression of 84 and 30\%, respectively, as determined from IHC staining intensity of $3+$ or 2+. This pattern of higher ERBB1 than ERBB2 expression

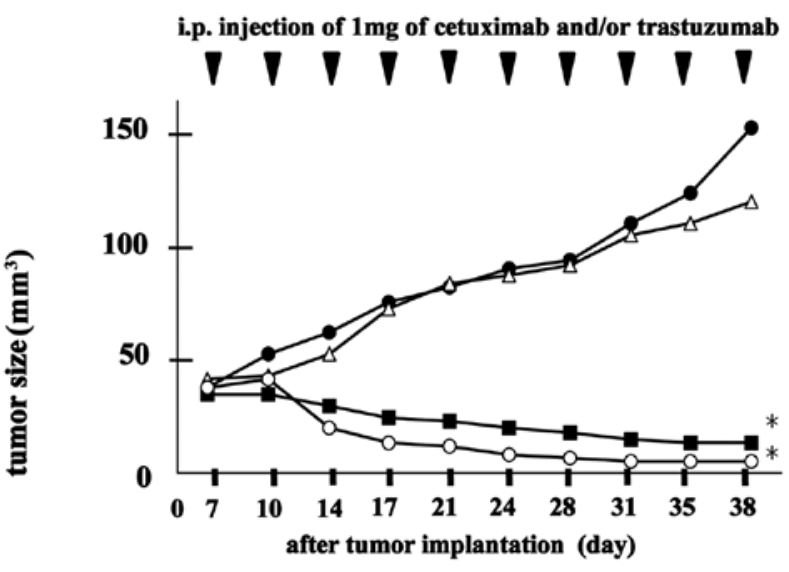

$* p<0.01$

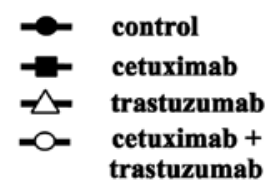

Figure 5. Antitumor effect of cetuximab and/or trastuzumab for TT cell line in vivo. In TT tumors, cetuximab, but not trastuzumab, revealed antitumor effect in vivo. In addition, combination of cetuximab and trastuzumab showed synergistic antitumor effect in vivo.

was also reflected in our flow cytometry and RT-PCR analysis. Previous studies have found a similar pattern of greater ERBB1 than ERBB2 expression, whereas others have found no ERBB2 expression in EC cells (13-17,19,23). Interestingly, it has also been suggested that expression of ERBB2 may vary at different stages of tumor progression (14).

ERBB1 expression, and to a lesser extent ERBB2 expression, has been correlated with tumor progression and prognosis in EC. Moreover, while the evidence suggests a strong association between ERBB1 expression and response to targeted therapy in EC, it is less well established in the case of ERBB2 $(13-15,17,18)$. Here, we found that cetuximab inhibited cellular proliferation in the EC cell lines while trastuzumab had no effect, but the combination of the two had a greater inhibitory effect than cetuximab alone. In similar studies, response to cetuximab and trastuzumab was generally consistent with the respective degree of expression of ERBB1 and ERBB2, and additive or synergistic effects of cetuximab plus trastuzumab in all samples except the high-ERBB2/low-ERBB1 TE4 cell line (16), and that cetuximab had a significant antiproliferative effect on high-ERBB1 expressing EC cell lines $(16,24)$. Further, Mimura et al (25) found marginal albeit still statistically significant anti-proliferative effects of trastuzumab on a variety of ERBB2-expressing EC cell lines. Sato et al (26) found that while trastuzumab alone had no effect on low-ERBB2 expressing EC cells, however, it did enhance radiosensitivity. While our study confirmed the anti-proliferative effects of cetuximab and combination therapy of cetuximab plus trastuzumab in ERBB1- and ERBB2-expressing cells, the role and extent of trastuzumab in inhibiting cellular proliferation in ERBB2-expressing EC cells remains unclear. 
One method by which monoclonal antibodies such as cetuximab and trastuzumab are thought to exert their anti-proliferative effect is via the induction of receptor downregulation from the cell surface $(21,22)$. Although this mechanism of action has been studied for both cetuximab and trastuzumab in other cancer types, to our knowledge the present study is the first to explored this mechanism in EC. Results showed that cetuximab induced ERBB1 downregulation whereas trastuzumab showed no effect on ERBB2 downregulation. In their review, Friedlander et al reported that although ERBB1 downregulation is an established mechanism of action for cetuximab, the ability of trastuzumab to downregulate ERBB2 and the role of this downregulation on the in vivo effects of trastuzumab is controversial (21). The respective role of cetuximab and trastuzumab in ERBB1 and ERBB2 downregulation in EC requires further study.

In conclusion, combination of cetuximab and trastuzumab revealed synergistic antitumor effect for squmous EC in vitro and in vivo. Then, the antitumor effect may be induced by the inhibition of phosphorylation of Akt. These findings suggest that combination therapy including cetuximab and trastuzumab may be promising strategy to treat squmous EC.

\section{References}

1. Enzinger PC and Mayer RJ: Esophageal cancer. N Engl J Med 349: 2241-2252, 2003.

2. Rice TW, Rusch VW, Apperson-Hansen C, et al: Worldwide esophageal cancer collaboration. Dis Esophagus 22: 1-8, 2009.

3. Jemal A, Siegel R, Ward E, et al: Cancer statistics, 2008. CA Cancer J Clin 58: 71-96, 2008.

4. De Vita F, Orditura M, Martinelli E, et al: A multicenter phase II study of induction chemotherapy with FOLFOX-4 and cetuximab followed by radiation and cetuximab in locally advanced oesophageal cancer. Br J Cancer 104: 427-432, 2011.

5. Lorenzen S, Schuster T, Porschen R, et al: Cetuximab plus cisplatin-5-fluorouracil versus cisplatin-5-fluorouracil alone in first-line metastatic squamous cell carcinoma of the esophagus: a randomized phase II study of the Arbeitsgemeinschaft Internistische Onkologie. Ann Oncol 20: 1667-1673, 2009.

6. Citri A and Yarden Y: EGF-ERBB signalling: towards the systems level. Nat Rev Mol Cell Biol 7: 505-516, 2006.

7. Ferguson KM: Structure-based view of epidermal growth factor receptor regulation. Annu Rev Biophys 37: 353-373, 2008.

8. Olayioye MA, Neve RM, Lane HA and Hynes NE: The ErbB signaling network: receptor heterodimerization in development and cancer. EMBO J 19: 3159-3167, 2000.

9. Normanno N, Bianco C, De Luca A, Maiello MR and Salomon DS: Target-based agents against ErbB receptors and their ligands: a novel approach to cancer treatment. Endocr Relat Cancer 10: 1-21, 2003.
10. Yarden Y and Sliwkowski MX: Untangling the ErbB signalling network. Nat Rev Mol Cell Biol 2: 127-137, 2001.

11. Citri A, Skaria KB and Yarden Y: The deaf and the dumb: the biology of ErbB-2 and ErbB-3. Exp Cell Res 284: 54-65, 2003.

12. Normanno N, De Luca A, Bianco C, et al: Epidermal growth factor receptor (EGFR) signaling in cancer. Gene 366: 2-16, 2006.

13. Morgan S and Grandis JR: ErbB receptors in the biology and pathology of the aerodigestive tract. Exp Cell Res 315: 572-582, 2009.

14. Ekman S, Bergqvist M, Heldin $\mathrm{CH}$ and Lennartsson J: Activation of growth factor receptors in esophageal cancer - implications for therapy. Oncologist 12: 1165-1177, 2007.

15. Ekman S, Dreilich M, Lennartsson J, et al: Esophageal cancer: current and emerging therapy modalities. Expert Rev Anticancer Ther 8: 1433-1448, 2008.

16. Kawaguchi Y, Kono K, Mimura K, et al: Targeting EGFR and HER-2 with cetuximab- and trastuzumab-mediated immunotherapy in oesophageal squamous cell carcinoma. Br J Cancer 97: 494-501, 2007.

17. Fiske WH, Threadgill D and Coffey RJ: ERBBs in the gastrointestinal tract: recent progress and new perspectives. Exp Cell Res 315: 583-601, 2009.

18. Lin CC and Papadopoulos KP: Novel targeted therapies for advanced esophageal cancer. Dis Esophagus 20: 365-371, 2007.

19. Wei Q, Chen L, Sheng L, Nordgren H, Wester K and Carlsson J: EGFR, HER2 and HER3 expression in esophageal primary tumours and corresponding metastases. Int J Oncol 31: 493-499, 2007.

20. Pande AU, Iyer RV, Rani A, et al: Epidermal growth factor receptor-directed therapy in esophageal cancer. Oncology 73: 281-289, 2007.

21. Friedlander E, Barok M, Szollosi J and Vereb G: ErbB-directed immunotherapy: antibodies in current practice and promising new agents. Immunol Lett 116: 126-140, 2008.

22. Schmitz KR and Ferguson KM: Interaction of antibodies with ErbB receptor extracellular regions. Exp Cell Res 315: 659-670, 2009.

23. Friess H, Fukuda A, Tang WH, et al: Concomitant analysis of the epidermal growth factor receptor family in esophageal cancer: overexpression of epidermal growth factor receptor mRNA but not of c-erbB-2 and c-erbB-3. World J Surg 23: 1010-1018, 1999.

24. Kawaguchi Y, Kono K, Mimura K, Sugai H, Akaike H and Fujii H: Cetuximab induce antibody-dependent cellular cytotoxicity against EGFR-expressing esophageal squamous cell carcinoma. Int J Cancer 120: 781-787, 2007.

25. Mimura K, Kono K, Hanawa M, et al: Trastuzumab-mediated antibody-dependent cellular cytotoxicity against esophageal squamous cell carcinoma. Clin Cancer Res 11: 4898-4904, 2005.

26. Sato S, Kajiyama Y, Sugano M, et al: Monoclonal antibody to HER-2/neu receptor enhances radiosensitivity of esophageal cancer cell lines expressing HER-2/neu oncoprotein. Int J Radiat Oncol Biol Phys 61: 203-211, 2005. 